\title{
MATHEMATICAL MODELLING OF ELASTIC DEFLECTION OF A TUBULAR CROSS-SECTION
}

\author{
Marcin ZASTEMPOWSKI, Ph.D. \\ Andrzej BOCHAT, Prof. \\ UTP University of Science and Technology, Poland
}

\begin{abstract}
Cutting a tubular element into thin rings is an operation often used in engineering processes. Total work of the cutting consists of that necessary for initial deflection of cut material and the cutting work itself. This paper presents mathematical models which describe deflection of cross-section of a single tubular element as well as a single layer of tubes under action of external forces (acting and reacting ones). The deflection concerns end part of tube hence the cutting object model has been reduced to a thin ring. Results of calculation by using the developed models are presented for selected materials of tubular elements, which are commonly used for structures of the kind; moreover, they are prepared in a form of dimensionless diagrams, that makes it possible to use them for an arbitrary material.
\end{abstract}

Keywords: mathematical modelling of elastic deflection, tubular elements, deflection of a ring under action of external forces

\section{INTRODUCTION}

Issues of elastic deflection of tubular cross-sections appear first of all in :

- load carrying structures of machines and devices, including ocean engineering objects,

- $\quad$ steel building structures,

- $\quad$ pipe-line installations, e.g. water supplying, heating and transporting ones,

- technological processes connected with breaking up tubular cross-sections by cutting, used e.g. in machine industry, plastic compound processing, agriculture cutting plant materials and their breaking up $[1,3,8]$.

In machinery construction area, are mainly considered issues dealing with design principles and strength analysis of steel structures [6], principles of FEM application and numerical analysis $[2,5,6]$, however detail issues concerning elastic deformations of structural elements are lacking in them.

One of the conditions for fast designing tubular structures or installations of the kind is the having at one's disposal adequate mathematical models describing process of their elastic deformation. In the subject-matter literature models which exactly describe process of elastic deformations of tubular elements under external load, especially in case where they mutually interact, are still lacking.

This work is hence aimed at the developing of mathematical models which describe deflection of a single tubular element
- a ring as well as a layer of rings under action of external forces.

\section{MATHEMATICAL MODEL OF ELASTIC DEFORMATIONS OF A SINGLE TUBULAR ELEMENT}

To the mathematical modelling of tubular element elastic deformations the calculation principle of curved bars, based on Castigliano's theorem, was applied and the following assumptions were made $[4,7]$ :

1. The tubular element is under action of the force $P$ and reaction $R$;

2. A single tubular element is taken as a ring of the Young modulus $E$ and inertia moment $I$;

3. Radius of neutral layer curvature is equal to the mean radius $r$ of the ring, calculated as follows :

$$
r=\frac{d_{w}+d_{z}}{2}
$$

where:

$d_{w}$-internal diameter of ring,

$d_{z}$ - external diameter of ring. 
Energy methods based on Castigliano's and Menabrea's theorems were applied to calculation of deformations $[4,7]$.

Let's consider the case in which a single tubular element is placed on stiff surface and loaded by the force $P$ (Fig.1).

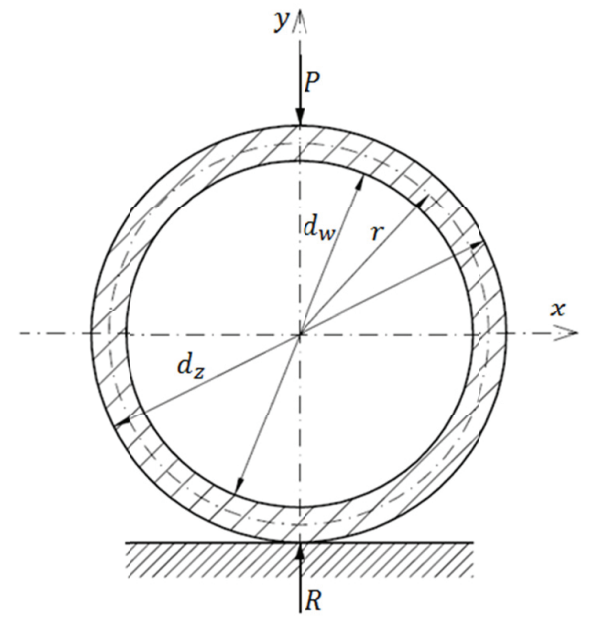

Fig.1. Cross-section of a thin ring under action of the force $P$

The ring loaded by the force $P$ and the support reaction $R$ is in state of equilibrium. To determine internal forces in the ring it is cut along horizontal axis $X$ into two symmetrical parts. The lower part of it is omit $\stackrel{P}{-}_{\mathrm{d}}$ and in places of cuts the reactions in the form of the force ${ }^{2}$ and bending moment $M_{0}$ are applied. The thus obtained system of forces acting on the upper part of the ring is shown in Fig. 2.

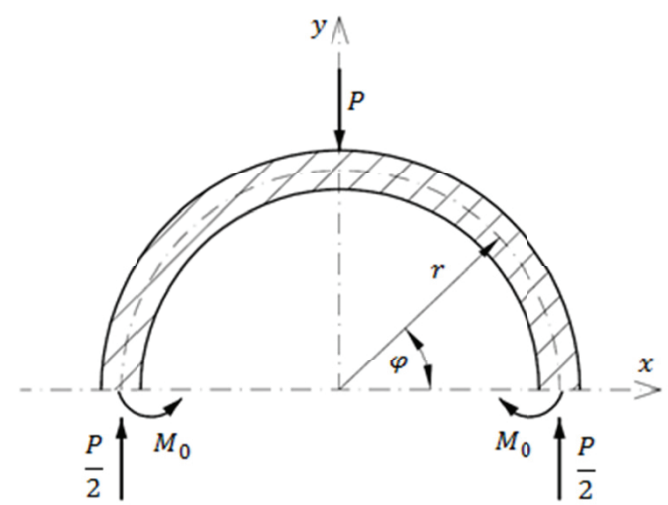

Fig. 2. The system of forces acting on the upper part of the ring

In the cross-section determined by the angle $\varphi$ in relation to the axis $x$ the following internal forces are acting (Fig.3):

- the force $N$ normal to the cross-section,

- the bending moment $M$.

In Fig. 3 the shear force $T=\frac{P}{2} \sin \varphi$ is not indicated as it does not appear in the relation (6) which describes the elastic strain energy $L$.

In order to determine internal forces a pair of $\mathrm{P} / 2$ equilibrating forces is introduced into the middle of the cross-section.

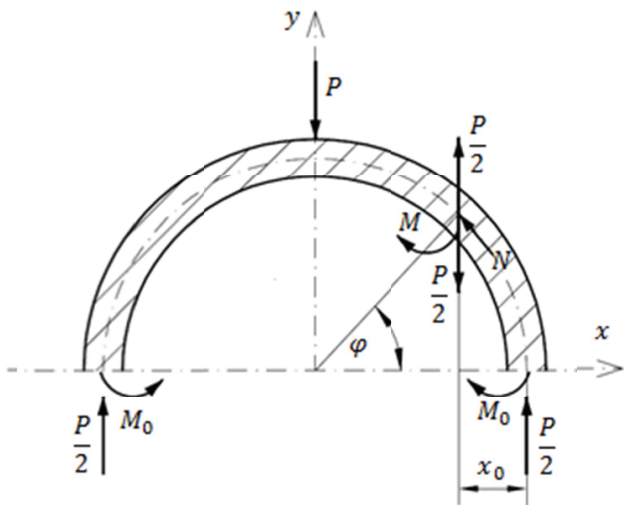

Fig. 3. The system of internal forces within the ring

The force $N$ can be then presented as follows :

$$
N=\frac{P}{2} \cos \varphi,
$$

and the internal moment $M$ - by using the relation :

$$
M=M_{0}-\frac{P}{2} x_{0} .
$$

Because:

$$
x_{0}=r(1-\cos \varphi) \text {, }
$$

the relation (3) takes the form:

$$
M=M_{0}-\frac{P}{2} r(1-\cos \varphi) \text {. }
$$

As the moment $M$ is hyper-static it will be determined by applying the Menabrea's theorem. The elastic strain energy $L$ accumulated in the ring is a sum of amounts of energy due to the bending moment $M$ and the compressive force $N[4,7]$ :

$$
L=\frac{1}{2} \int_{0}^{s} \frac{M^{2}}{E J} d s+\frac{1}{2} \int_{0}^{s} \frac{N^{2}}{E F} d s,
$$

where:

$N$-compressive force,

$M$ - bending moment,

$E$ - Young modulus,

I - inertia moment,

$F$ - ring cross-section area,

$s-$ arc length of deformed ring,

$d s$ - elementary length of ring arc $: d s=r d \varphi$,

$\varphi$ - cross-section location angle,

$d \varphi$ - elementary angle of $\varphi$. 
By introducing a polar coordinate system, i.e. $d s=r d \varphi$ the energy contained in a quarter of ring takes the form :

$$
L_{\frac{1}{4}}=\frac{1}{2} \int_{0}^{\frac{\pi}{2}} \frac{M^{2}}{E J} r d \varphi+\frac{1}{2} \int_{0}^{\frac{\pi}{2}} \frac{N^{2}}{E F} r d \varphi .
$$

Energy for the whole ring, due to its symmetry, amounts to :

$$
L=4 L_{\frac{1}{4}}=\frac{2 r}{E J} \int_{0}^{\frac{\pi}{2}} M^{2} d \varphi+\frac{2 r}{E F} \int_{0}^{\frac{\pi}{2}} N^{2} d \varphi .
$$

In compliance with Menabrea's theorem the partial derivative of elastic strain energy of a system in relation to statically indeterminate reaction is equal to zero $[4,7]$ :

$$
\frac{\partial L}{\partial M_{0}}=0
$$

The relation (9) which makes it possible to determine the moment $\mathrm{M}_{0}$, takes, after transformation, the following form :

$$
\frac{\partial L}{\partial M_{0}}=\frac{4 r}{E J} \int_{0}^{\frac{\pi}{2}} M \frac{\partial M}{\partial M_{0}} d \varphi+\frac{4 r}{E F} \int_{0}^{\frac{\pi}{2}} N \frac{\partial N}{\partial M_{0}} d \varphi=0 .
$$

Taking into account (2) and (3) one obtains :

$$
\frac{\partial M}{\partial M_{0}}=1 \text { and } \frac{\partial N}{\partial M_{0}}=0
$$

The equation (10) may be then presented as follows :

$$
\frac{\partial L}{\partial M_{0}}=\frac{4 r}{E J} \int_{0}^{\frac{\pi}{2}} M d \varphi=0 .
$$

On substitution of the relation (5) into the equation (11) and simplification the following is obtained:

$$
\int_{0}^{\frac{\pi}{2}}\left[M_{0}-\frac{P}{2} r(1-\cos \varphi)\right] d \varphi=0,
$$

which after integration gives :

$$
\left[M_{0} \varphi-\frac{P}{2} r \varphi+\frac{P}{2} r \sin \varphi\right]_{0}^{\frac{\pi}{2}}=0
$$

When integration limits are taken into account and reduction is made the following is reached:

$$
M_{0}=\left(\frac{1}{2}-\frac{1}{\pi}\right) \operatorname{Pr} \text {. }
$$

As the expression for the moment $M_{0}$ is known, the equation for the bending moment $M$ in the ring, resulting from the substitution of the expression (14) into the relation (5), takes the form :

$$
M=\left(\frac{\cos \varphi}{2}-\frac{1}{\pi}\right) P r
$$

As the expression which describes bending moment is known, deflection of the ring under action of the force $P$ can be obtained by making use of the Castigliano's theorem. To this end, partial derivative of the elastic strain energy $L$ in relation to the force $P$ is calculated.

$$
f=\frac{\partial L}{\partial P}=\frac{4 r}{E J} \int_{0}^{\frac{\pi}{2}} M \frac{\partial M}{\partial P} d \varphi+\frac{4 r}{E F} \int_{0}^{\frac{\pi}{2}} N \frac{\partial N}{\partial P} d \varphi .
$$

By taking into account :

$$
\frac{\partial M}{\partial P}=\left(\frac{\cos \varphi}{2}-\frac{1}{\pi}\right) r
$$

and :

$$
\frac{\partial N}{\partial P}=\frac{1}{2} \cos \varphi,
$$

the following is obtained:

$f=-\frac{4 r}{E J} \int_{0}^{\frac{\pi}{2}} \operatorname{Pr}^{2}\left(\frac{\cos \varphi}{2}-\frac{1}{\pi}\right)^{2} d \varphi+\frac{4 r}{E F} \int_{0}^{\frac{\pi}{2}} \frac{P}{2} \cos \varphi \frac{1}{2} \cos \varphi d \varphi$.

On its integration, the elastic deflection of a ring - form tubular element under action of the force $P$, is equal to :

$$
f=\left(\frac{\pi}{4}-\frac{2}{\pi}\right) \frac{P r^{3}}{E J}+\frac{\pi}{4} \frac{P r}{E F}
$$

The total deflection $f$ is connected with direct action of the force $N$ and the bending moment $M$. Therefore the total deflection of tubular element cross-section can be described as follows:

$$
f=f_{1}+f_{2}
$$

where:

$f_{1}$ - deflection due to action of the bending moment $M$,

$f_{2}$ - deflection due to action of the compressive force $N$.

By taking into account the equations (18) and (19) the expressions for the deflections $f_{1}$ and $f_{2}$ take the following forms, respectively:

$$
f_{1}=\left(\frac{\pi}{4}-\frac{2}{\pi}\right) \frac{P r^{3}}{E J}
$$

and

$$
f_{2}=\frac{\pi}{4} \frac{P r}{E F}
$$

As the mean radius of the ring, $r$, ring cross-section inertia modulus $I$ and cross-section area $F$ are functions of the ratio of ring diameters,$\frac{d_{w}}{d_{z}}$, results of ring deformation 
calculations will be presented in the dimensionless form $\bar{f}$. Actual deflection of a ring depends also on its thickness $b$ and amounts to:

$$
f=\bar{f} \frac{P}{E b},
$$

where:

$$
\bar{f}=\bar{f}_{1}+\bar{f}_{2}
$$

Fig. 4 presents a diagram of dimensionless deflection of a single ring element in function of the ratio of tubular crosssection diameters.

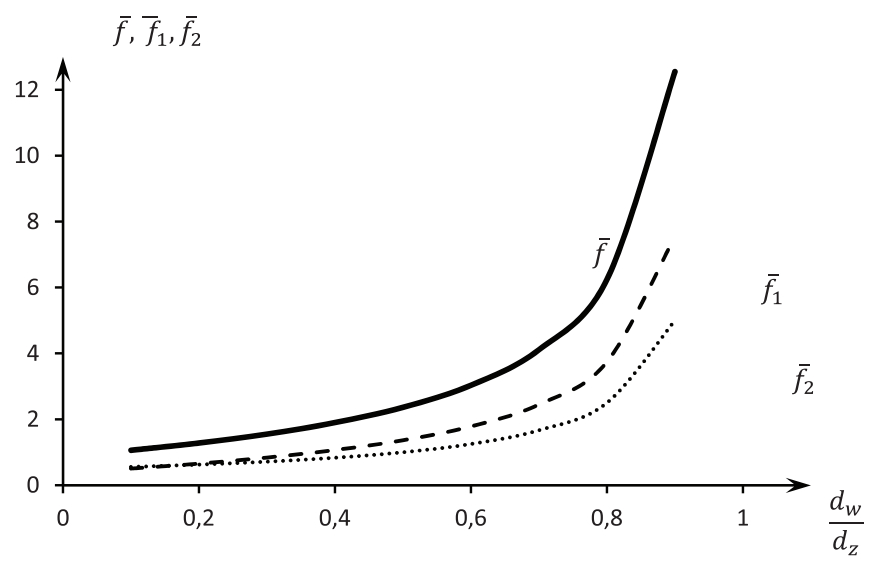

Fig. 4. Dimensionless deflection of a single ring element under action of the force $P$ : $\bar{f}$ - relative total deflection, $\bar{f}_{1}$ - relative deflection resulting from the bending moment $M, f_{2}$-relative deflection resulting from the compressive force $N, d_{w^{-}}$ring element internal diameter, $d_{z^{-}}$ring element external diameter

From the above presented diagram it results that for the values of the diameters ratio of a single tubular element, contained in the range of $0,1 \div 0,9$ the relative deformation of its cross-section varies approximately linearly, and for the diameters ratio above 0,8 the change departs from linearity and grows fast ( approximately exponentially ). Run of particular curves is similar while the deflection resulting from action of the bending moment $M$ takes greater values than that due to action of the compressive force $N$ at given ratios of tubular element diameters Actual deflection of analysed element depends on the force $P$, Young modulus $E$ and ring thickness $b$, that is expressed by the relation (22).

\section{MATHEMATICAL MODEL OF ELASTIC DEFLECTION OF A LAYER OF TUBULAR ELEMENTS}

For the layer of tubular elements it is assumed that they mutually interact and their transverse deflection equals zero (Fig.5). The elements are placed in a stiff casing, free of compression and gaps between them.

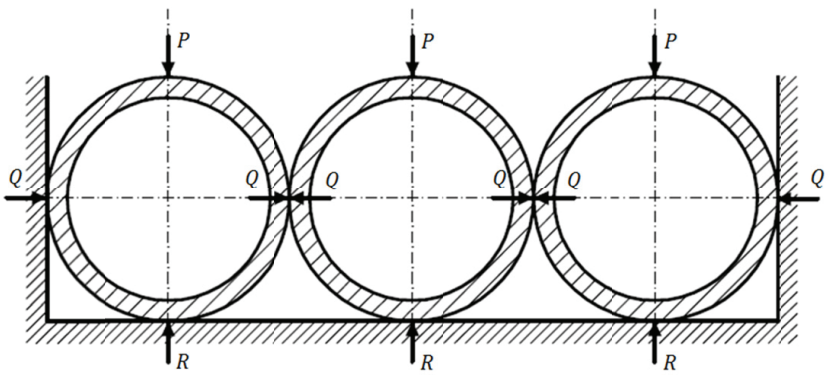

Fig. 5. Layer of ring tubular elements loaded by vertical forces $P$

In order to solve such a system the model presented in Fig. 6 was assumed, in which the additional force $Q$ resulting from interaction of neighbouring elements was taken into account.

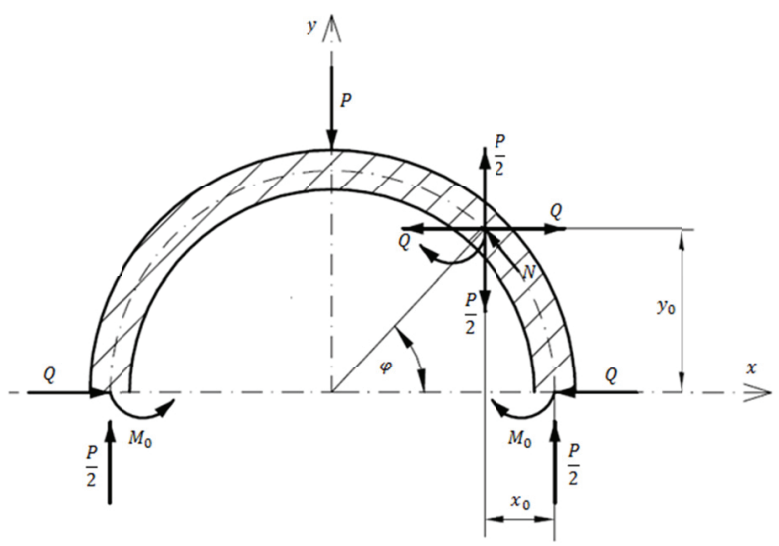

Fig. 6. The system of forces acting onto the upper part of the ring

In the cross-section determined by the angle $\varphi$ in relation to $x$ - axis the following internal loads are acting (Fig. 6):

- the compressive force $N$,

- the bending moment $M$.

In order to determine the internal loads two pairs of equilibrating forces, $\frac{-}{2}$ and $Q$, are introduced into the middle of the cross-section.

The force $N$ in an arbitrary cross-section may be then described as follows :

$$
N=\frac{P}{2} \cos \varphi+Q \sin \varphi
$$

a $\mathrm{n} \mathrm{d}$

the bending moment $M$ - by the relation:

$$
M=M_{0}-\frac{P}{2} x_{0}+Q y_{0}
$$

As:

$$
x_{0}=(1-\cos \varphi) r \text { and } y_{0}=r \sin \varphi \text {, }
$$

hence, finally, the moment $M$ is described by the relation:

$$
M=M_{0}-\frac{P}{2} r(1-\cos \varphi)+Q r \sin \varphi .
$$


Because the moment $M_{0}$ and the force $Q$ are hyper-static they were determined by using the Menabrea's theorem [4, 7]:

$$
\frac{\partial L}{\partial M_{0}}=0 \quad \text { oraz } \quad \frac{\partial L}{\partial Q}=0
$$

As a result, the following was obtained:

$$
\frac{\partial L}{\partial M_{0}}=\frac{4 r}{E J} \int_{0}^{\frac{\pi}{2}} M \frac{\partial M}{\partial M_{0}} d \varphi+\frac{4 r}{E F} \int_{0}^{\frac{\pi}{2}} N \frac{\partial N}{\partial M_{0}} d \varphi=0
$$

and:

$$
\frac{\partial L}{\partial Q}=\frac{4 r}{E J} \int_{0}^{\frac{\pi}{2}} M \frac{\partial M}{\partial Q} d \varphi+\frac{4 r}{E F} \int_{0}^{\frac{\pi}{2}} N \frac{\partial N}{\partial N} d \varphi=0 .
$$

The partial derivatives appearing in the equations (27) and (28) obtain the following values:

$\frac{\partial M}{\partial M_{0}}=1, \quad \frac{\partial M}{\partial Q}=r \sin \varphi, \quad \frac{\partial N}{\partial M_{0}}=0, \quad \frac{\partial N}{\partial Q}=\sin \varphi$.

On their respective introduction to the equations (27) and (28) the following was reached:

$$
\frac{\partial L}{\partial M_{0}}=\frac{4 r}{E J} \int_{0}^{\frac{\pi}{2}} M d \varphi=0,
$$

and

$$
\frac{\partial L}{\partial Q}=\frac{4 r}{E J} \int_{0}^{\frac{\pi}{2}} M r \sin \varphi d \varphi+\frac{4 r}{E F} \int_{0}^{\frac{\pi}{2}} N \sin \varphi d \varphi=0 .
$$

By respective introducing the equations (24) and (26) into the equations (22) i (23) and then integrating and reducing them, the following system of equations was achieved :

$$
\left\{\begin{array}{l}
\frac{M_{0}}{r}+\frac{\pi}{4} Q(1+\delta)+\frac{P}{4}(\delta-1)=0, \\
\frac{\pi}{2} \frac{M_{0}}{r}+Q-\frac{P}{2}\left(\frac{\pi}{2}\right)-1=0,
\end{array}\right.
$$

where the factor $\delta$ is equal to:

$$
\delta=\frac{J}{F r^{2}}
$$

As a result of solving the above presented system of equations the following was obtained:

$$
M_{0}=\frac{\left(\frac{\pi^{2}}{2}-\pi\right)(1+\delta)+2(\delta-1)}{\pi^{2}(1+\delta)-8} \operatorname{Pr}
$$

By assuming that:

$$
\gamma=\frac{\left(\frac{\pi^{2}}{2}-\pi\right)(1+\delta)+2(\delta-1)}{\pi^{2}(1+\delta)-8} .
$$

the equation (34) takes the form:

$$
M_{0}=\gamma P r
$$

As a result of solving the system of the equations (31) and (32) the relation for $Q$ was obtained:

$$
Q=\frac{\pi(1-\delta)+4-2 \pi}{\pi^{2}(1+\delta)-8} P
$$

By assuming that:

$$
\alpha=\frac{\pi(1-\delta)+4-2 \pi}{\pi^{2}(1+\delta)-8},
$$

the relation (37) takes the form:

$$
Q=\alpha P
$$

As the expressions describing the bending moment $M_{0}$ and the force $Q$ are already known, it is possible to determine the relation for the bending moment $M$ by introducing the relations (36) and (37) into the expression (26) :

$$
M=\operatorname{Pr}\left(\gamma-\frac{1}{2}+\frac{1}{2} \cos \varphi+\alpha \sin \varphi\right)
$$

In order to determine deflection of the ring under action of the force $P$ the Castigliano's theorem should be used by calculating the par ${ }_{P}^{+}$al derivative of the elastic strain energy $L$ against the force $P$. Hence:

$$
f=\frac{\partial L}{\partial P}=\frac{4 r}{E J} \int_{0}^{\frac{\pi}{2}} M \frac{\partial M}{\partial P} d \varphi+\frac{4 r}{E F} \int_{0}^{\frac{\pi}{2}} N \frac{\partial N}{\partial P} d \varphi
$$

The total deflection $f$ is associated with common action the compressive force $N$ and the bending moment $M$. Hence:

$$
f=f_{1}+f_{2}
$$

where:

$f_{1}$ - deflection resulting from the bending moment $M$, $f_{2}$-deflection resulting from the compressive force $N$. 
Therefore :

$$
f_{1}=\frac{4 r}{E J} \int_{0}^{\frac{\pi}{2}} M \frac{\partial M}{\partial P} d \varphi
$$

and:

$$
f_{2}=\frac{4 r}{E F} \int_{0}^{\frac{\pi}{2}} N \frac{\partial N}{\partial P} d \varphi
$$

By taking into account the relation:

$$
\frac{\partial M}{\partial P}=r\left(\gamma-\frac{1}{2}+\frac{1}{2} \cos \varphi+\alpha \sin \varphi\right)
$$

and :

$$
\frac{\partial N}{\partial P}=\frac{1}{2} \cos \varphi+\alpha \sin \varphi
$$

the following is obtained:

$f_{1}=\frac{4 \operatorname{Pr}^{3}}{E J} \int_{0}^{\frac{\pi}{2}}\left(\gamma-\frac{1}{2}+\frac{1}{2} \cos \varphi+\alpha \sin \varphi\right)^{2} d \varphi$.

And, $f_{2}$ is described by the relation :

$$
f_{2}=\frac{4 P r}{E F} \int_{0}^{\frac{\pi}{2}}\left(\frac{1}{2} \cos \varphi+\alpha \sin \varphi\right)^{2} d \varphi .
$$

The equations (44) and (45) may be presented in the form :

$$
\begin{gathered}
f_{1}=\frac{4 P r^{3}}{E J} g_{1}, \\
f_{2}=\frac{4 P r}{E F} g_{2},
\end{gathered}
$$

by assuming that:

$$
\begin{gathered}
g_{1}=\int_{0}^{\frac{\pi}{2}}\left(\gamma-\frac{1}{2}+\frac{1}{2} \cos \varphi+\alpha \sin \varphi\right)^{2} d \varphi, \\
g_{2}=\int_{0}^{\frac{\pi}{2}}\left(\frac{1}{2} \cos \varphi+\alpha \sin \varphi\right)^{2} d \varphi .
\end{gathered}
$$

In view of the complex form of the equations (48) and (49) the factors $g_{1}$ and $g_{2}$ were determined by numerical calculations whose results are presented in Tab. 1 .

Tab. 1. Results of calculation of the factors $g_{1}$ and $g_{2}$

\begin{tabular}{|c|c|c|c|c|}
\hline$\frac{d_{w}}{d_{z}}$ & $\mathbf{0 , 1}$ & $\mathbf{0 , 2}$ & $\mathbf{0 , 3}$ & $\mathbf{0 , 4}$ \\
\hline$g_{1}$ & 0,0305 & 0,0273 & 0,0248 & 0,0231 \\
\hline$g_{2}$ & 0,2243 & 0,2412 & 0,2553 & 0,2664 \\
\hline
\end{tabular}

\begin{tabular}{|c|c|c|c|c|c|}
\hline$\frac{d_{w}}{d_{z}}$ & $\mathbf{0 , 5}$ & $\mathbf{0 , 6}$ & $\mathbf{0 , 7}$ & $\mathbf{0 , 8}$ & $\mathbf{0 , 9}$ \\
\hline$g_{1}$ & 0,0219 & 0,0211 & 0,0206 & 0,0203 & 0,0200 \\
\hline$g_{2}$ & 0,2748 & 0,2808 & 0,2849 & 0,2874 & 0,2887 \\
\hline
\end{tabular}

In Fig. 7 is presented a diagram of dimensionless deflection of a ring tubular element layer in function of the ratio of tubular element diameters.

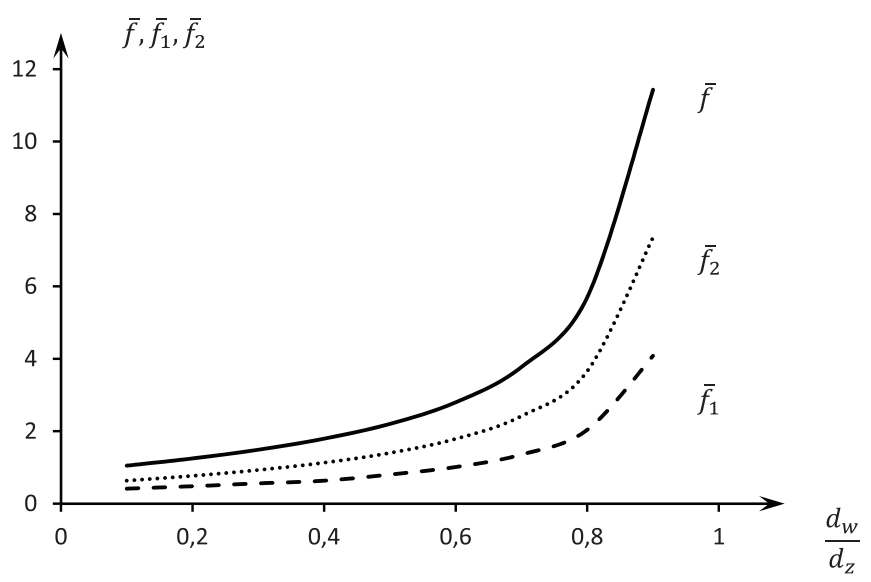

Fig. 7. Dimensionless deflection of ring tubular elements placed in a layer under action of the force $P: f$-relative total deflection, $f_{1}$ - relative deflection resulting from the bending moment $M, f_{2}$-relative deflection resulting from the compressive force $N, d_{w^{-}}$tubular element internal diameter, $d_{z}$-tubular element external diameter

From the above presented diagram it results that for the values of the ratio of diameters of tubular elements placed in a layer, contained in the range of $0,1 \div 0,9$ the relative deformation of their cross-sections varies approximately linearly, and for the diameters ratio above 0,8 the change departs from linearity and grows fast ( approximately exponentially). Run of particular curves is similar and - in contrast to a single element - the deflection of tubular element layer due to action of the compressive force $N$ takes greater values than that resulting from action of the bending moment $M$ at given ratios of tubular element diameters. Actual deflection of the analysed tubular element system depends on the force $P$, Young modulus $E$ and diameter $d_{z}$.

Exemplary calculations of deformations of tubular element cross-sections were additionally performed for selected 
materials. In the calculations the tubular elements of commercial dimensions characteristic for a given material ,were considered.

Tab. 2 presents calculation results of deflection of a single tubular element and a layer of tubular elements under action of external forces , made of steel of the Young modulus $E=$ $210 \mathrm{GPa}$, under action of the force $P=100 \mathrm{~N}$. The calculations were performed for the element thickness $b=10 \mathrm{~mm}$.

Tab. 2. Values of deflection of tubular elements made of steel

\begin{tabular}{|c|c|c|c|}
\hline \multirow[b]{2}{*}{ No. } & \multirow{2}{*}{$\begin{array}{c}\text { Element } \\
\text { dimensions }\end{array}$} & \multicolumn{2}{|c|}{ Deflection $f[\mu \mathrm{m}]$} \\
\hline & & $\begin{array}{c}\text { Single } \\
\text { element }\end{array}$ & $\begin{array}{c}\text { Tubular } \\
\text { elements layer }\end{array}$ \\
\hline 1 & $1 / 4 "(13,5 \times 2,35)$ & 1,22 & 0,43 \\
\hline 2 & $1 / 2 ” \quad(21,3 \times 2,65)$ & 3,84 & 0,97 \\
\hline 3 & $3 / 4 " \quad(26,9 \times 2,65)$ & 8,32 & 1,78 \\
\hline 4 & $1 " \quad(33,7 \times 3,25)$ & 8,92 & 1,89 \\
\hline 5 & $11 \frac{1}{2} "(48,3 \times 3,25)$ & 28,56 & 5,21 \\
\hline
\end{tabular}

Tab. 3 presents calculation results of deflection of a single tubular element and a layer of tubular elements under action of external forces, made of copper of the Young modulus $E=$ $110 \mathrm{GPa}$, under action of the force $P=100 \mathrm{~N}$. The calculations were performed for the element thickness $b=10 \mathrm{~mm}$.

Tab. 3. Values of deflection of tubular elements made of copper

\begin{tabular}{|c|l|c|c|}
\hline \multirow{2}{*}{ No. } & \multirow{2}{*}{$\begin{array}{c}\text { Element } \\
\text { dimensions }\end{array}$} & $\begin{array}{c}|c| \\
\text { Single } \\
\text { element }\end{array}$ & $\begin{array}{c}\text { Tubular } \\
\text { elements layer }\end{array}$ \\
\hline 1 & $10^{\mathrm{x}} 1$ & 15,11 & 3,27 \\
\hline 2 & $12^{\mathrm{x}} 1$ & 27,40 & 5,40 \\
\hline 3 & $15^{\mathrm{x}} 1$ & 56,17 & 10,22 \\
\hline 4 & $18^{\mathrm{x}} 1$ & 100,3 & 17,45 \\
\hline 5 & $22^{\mathrm{x}} 1$ & 188,6 & 31,71 \\
\hline
\end{tabular}

Tab. 4 presents calculation results of deflection of a single tubular element and a layer of tubular elements under action of external forces, made of aluminium of the Young modulus $E=70 \mathrm{GPa}$, under action of the force $P=100 \mathrm{~N}$. The calculations were performed for the element thickness $b=10 \mathrm{~mm}$.

Tab. 4. Values of deflection of tubular elements made of aluminium

\begin{tabular}{|c|l|c|c|}
\hline \multirow{2}{*}{ No. } & \multirow{2}{*}{$\begin{array}{c}\text { Element } \\
\text { dimensions }\end{array}$} & $\begin{array}{c}\text { Single } \\
\text { element }\end{array}$ & $\begin{array}{c}\text { Tubular } \\
\text { elements layer }\end{array}$ \\
\cline { 3 - 4 } & & 4,27 & 1,43 \\
\hline 1 & $6^{\mathrm{x}} 1$ & 11,33 & 2,86 \\
\hline 2 & $8^{\mathrm{x}} 1$ & 23,75 & 5,14 \\
\hline 3 & $10^{\mathrm{x}} 1$ & 43,05 & 8,49 \\
\hline 4 & $12^{\mathrm{x}} 1$ & 88,27 & 16,06 \\
\hline 5 & $15^{\mathrm{x}} 1$ & & \\
\hline
\end{tabular}

\section{SUMMARY}

The mathematical models presented in this work make it possible to carry out simulation of the process of elastic deflection of tubular elements in the form of rings. It has been proved that, according to the developed model, mutual interaction of elements ( rings ) placed in a layer significantly influences values of obtained deflections. It can be read from the results of simulation calculations presented in this work. The results are given in a form of dimensionless diagrams as well as in the tables containing values of deflections of tubular element ( ring) cross-sections, calculated for selected commercial dimensions and materials.

After positive experimental verification, the developed models would be ready for application in design stage of selected structures.

\section{BIBLIOGRAPHY}

1. Bochat A., Grzonkowski R., Zastempowski M.: Analysis of plant stalk cutting and a novel cutting drum unit (in Polish ). Inżynieria i Aparatura Chemiczna, No.1-2/2005.

2. Bohdal 1., Kukiełka L.: Application of variational and FEM methods to the modelling and numerical analysis of guillotining process for geometrical and physical nonlinearity. Mechanika vol. 20, No. 2, 2014. pp. 197-204.

3. Chattopahyay P.S., Pandey K.P.: Mechanical properties of sorghum in relation to quasi- static deformation. Journal of Agricultural Engineering Research. Vol.73, 1999.

4. Jakubowicz A, Orłoś Z.: Strength of materials (in Polish ). WNT Publishers, Warsaw, 1999.

5. Malag L, Kukiełka L.: The sensitivity analysis of influence of chosen material parameters on the properties of surface layer product after burnishing. Steel Research International. Volume SPL. ISSUE, 2012, pp. 1335-1338.

6. Malag L.,Kukielka L., Kukielka K., Kulakowska A., Bohdal L., Patyk R.: Problems determining of the mechanical properties of metallic materials from the tensile test in the aspect of numerical calculations of the technological 
processes. Applied Mechanics and Materials. Vol. 474, 2014, pp. 454-459

7. Niezgodziński M.E., Niezgodziński T.: Strength of materials ( in Polish ). PWN Publishers, Warsaw, 1984.

8. O’Doherty M.J., Huber J.A., Dyson J., Marshall C.J.: A study of the physical and mechanical properties of wheat straw. Journal of Agricultural Engineering Research, Vol.62, 1995.

9. Szala G. Comments on linear summation hypothesis of fatigue failures. Polish Maritime Research. Nr 3 (83) Vol.21, 2014. pp. 77-85.

\section{CONTACT WITH THE AUTHOR}

Marcin ZASTEMPOWSKI, Andrzej BOCHAT

UTP University of Science and Technology

20 Ks. Kordeckiego St. 85-225 Bydgoszcz

Poland 